OPEN ACCESS

Edited by:

Can Ozan Tan,

Harvard Medical School,

United States

Reviewed by:

Hidetaka Hamasaki, Hamasaki Clinic, Japan

Stacey E. Aaron,

Harvard Medical School,

United States

*Correspondence: Iztok Štotl

iztok.stot@@guest.arnes.si

Specialty section:

This article was submitted to

Exercise Physiology,

a section of the journal

Frontiers in Physiology

Received: 23 May 2019 Accepted: 05 September 2019 Published: 24 September 2019

Citation:

Štotl I, Kambič T, Hadžić $V$ and Zdolšek A (2019) Different Types of Physical Activity and Metabolic

Control in People With Type 1

Diabetes Mellitus.

Front. Physiol. 10:1210.

doi: 10.3389/fphys.2019.01210

\section{Different Types of Physical Activity and Metabolic Control in People With Type 1 Diabetes Mellitus}

\author{
Iztok Štot|1*, Tim Kambič̌̌,3, Vedran Hadžićc and Anže Zdolšek ${ }^{2}$ \\ ${ }^{1}$ Department of Endocrinology, Diabetes and Metabolic Diseases, University Medical Centre Ljubljana, Ljubljana, Slovenia, \\ ${ }^{2}$ Faculty of Sport, University of Ljubljana, Ljubljana, Slovenia, ${ }^{3}$ Department for Research and Education, General Hospital \\ Murska Sobota, Murska Sobota, Slovenia
}

Background: The aim of presented cross-sectional study was to determine the association of different types of physical activity (PA) with metabolic control in people with type 1 diabetes.

Materials and Methods: A total of 109 adult subjects with type 1 diabetes were asked to complete the non-exercise activity thermogenesis (NEAT) questionnaire, the hypoglycemia questionnaire, and the World Health Organization Global PA Questionnaire (GPAQ) which was used to assess moderate PA (MPA) and vigorous PA (VPA).

Results: NEAT score $(p<0.001)$ and total duration of work as assessed with GPAQ $(p=0.007)$ were positively associated with chronic glycemic control when controlled for sex, BMI, and continuous glucose monitoring system (CGMS) use. We could not confirm such association with total leisure time PA (LTPA) assessed with GPAQ ( $p=0.443$ ), though. Multivariate regression model controlled for sex showed positive effects of $\mathrm{HbA}_{1 c}(p=0.011)$ and age $(p=0.035)$, and negative effect of NEAT score $(p=0.001)$ on BMI. Systolic blood pressure was positively associated with duration of MPA $(p=0.009)$ and VPA $(p=0.012)$, but not with NEAT score $(p=0.830)$ when controlled for sex and BMI. NEAT score and VPA were positively associated with HDL levels when controlled for sex and BMI. Controlled for sex and BMI, higher values of VPA were significantly associated with lower levels of total cholesterol $(p=0.009)$ and LDL $(p=0.005)$.

Conclusion: Higher levels of NEAT are associated with some favorable metabolic effects in adult people with type 1 diabetes, but may also present an additional burden for them with more challenging environment regarding glycemic control.

Keywords: physical activity, type 1 diabetes, obesity, hypoglycemia, metabolism

Abbreviations: BMI, body mass index; CGMS, continuous glucose monitoring system; DBP, diastolic blood pressure; GPAQ, global physical activity questionnaire; HDL, high-density lipoprotein; LDL, low-density lipoprotein; LTPA, leisure time physical activity; MDI, multiple daily injections; MPA, moderate physical activity; NEAT, non-exercise activity thermogenesis; NEPA, non-exercise physical activity; PA, physical activity; SBP, systolic blood pressure; VPA, vigorous physical activity. 


\section{INTRODUCTION}

Physical activity has an important role in many aspects of treatment of people with type 1 diabetes (Riddell et al., 2017). Recent meta-analysis did not show benefit of exercise as measured by $\mathrm{HbA}_{1 c}$ (Ostman et al., 2018), while other studies show inconsistent results (Chimen et al., 2012). Large cross-sectional study of 18,028 adults with type 1 diabetes showed that people who engaged more in recreational exercise had better $\mathrm{HbA}_{1 c}$ concentrations, a more favorable $\mathrm{BMI}$, less dyslipidemia and hypertension, and fewer diabetesrelated complications (retinopathy and microalbuminuria) than those who were less habitually active (Bohn et al., 2015). A 7-year prospective cohort analysis performed in 1,659 people with type 1 diabetes could not find association between $\mathrm{HbA}_{1 c}$ levels and amount of LTPA (Balk et al., 2016). In another cross-sectional study of 1,030 people with type 1 diabetes low levels of LTPA were associated with poor glycemic control in women, but there was no statistical association with levels of LTPA and $\mathrm{HbA}_{1 c}$ in men (Wadén et al., 2005).

Physical activity can be divided into structured PA and NEPA. NEPA is distinct from purposeful exercise and includes every PA that is not sleeping, eating, or sports-like exercise and includes various activities in daily life such as going to work, attending school, singing, dancing, washing clothes, cleaning floor, etc. NEAT represents the energy expenditure associated with these activities (Levine, 2004). The variety of different types of activity that composes total NEPA creates a challenge for assessment of NEAT. Researchers have tried to estimate NEPA/NEAT with the use of double labeled water, indirect calorimetry, accelerometer, pedometer, inclinometer, heart rate, and activity diary (Silva et al., 2018).

Amount of NEPA affects daily glucose excursions (Ogata et al., 2013). Interrupting sitting time with short bouts of light or moderate intensity walking lowers postprandial glucose and insulin levels in overweight/obese adults (Dunstan et al., 2012). In people with type 1 diabetes, slow-pace walking after a meal improves postprandial glucose excursions when observed in controlled study environment (Manohar et al., 2012). Routine daily PAs of everyday life seem to be coupled with glucose variations in type 1 diabetes (Farabi et al., 2015). Recent study has successfully provided quantitative information on correlation between mild PA and short-term glucose dynamics in type 1 diabetes (Zecchin et al., 2013), that could be used in future models to improve glucose management with combination of advanced sensor technologies (Ding and Schumacher, 2016).

Majority of research that tries to study effects of PA on metabolic control in people with type 1 diabetes assesses predominantly planned exercise or LTPA. While higher levels of NEAT are known to be associated with many health benefits in people with type 2 or without diabetes (Levine, 2015), long-term metabolic effects of NEAT in people with type 1 diabetes have not been elucidated, yet. The aim of this study was to determine the association between different types of PA and metabolic control in people with type 1 diabetes.

\section{MATERIALS AND METHODS}

\section{Sample and Study Protocol}

One hundred and nine adult people with documented type 1 diabetes, that agreed to participate in the study and signed a written consent, were consecutively enrolled in the study on their regular visit at outpatient diabetes clinic in University Medical Centre Ljubljana (from January to March 2018). People with substantial limitations for everyday PA or pregnancy were excluded from the study. One homeless person was also excluded, because his answers would be inappropriate for NEAT questionnaire in current form.

During the regular visits, body weight was measured using Seca 285 device (Seca GmbH, Germany), while blood pressure was obtained using automatic sphygmomanometer Omron M6 (Omron, Osaka, Japan). Blood samples were withdrawn for $\mathrm{HbA}_{1 c}$ analysis using D-100 Bio-Rad high-performance liquid chromatography method (Bio-Rad Laboratories, United States), whereas serum lipid profile was retrieved from electronic health record, if they were collected routinely within 1 year before inclusion (available for 75 participants). The study protocol was approved by the National Medical Ethics Committee of Slovenia (Ref. No. 0120-258/2017/4).

Participants were asked to complete World Health Organization GPAQ (Armstrong and Bull, 2006) for assessment of MPA and VPA at work (such as paid or unpaid work, study/training, household chores, harvesting food/crops, fishing or hunting for food, and seeking employment), during traveling to and from places and during recreational activities. Duration of VPA per week in minutes (VPA) was calculated with the sum of VPA at work and recreation, whereas duration of MPA per week in minutes (MPA) was calculated with the sum of MPA at work, recreation, and transport.

Non-exercise activity thermogenesis was assessed with the questionnaire that was developed by Hamasaki et al. (2013) for assessment of NEAT in people with type 2 diabetes. The NEAT questionnaire consists of 11 question items about daily locomotive activities (about commuting on foot, shopping for food, using stairs, etc.) and 25 question items about nonlocomotive activities (being active in different domestic chores, looking after children, elderly, or pets, etc.). Prior to NEAT questionnaire administration, question regarding the karaoke singing was changed to choir singing, which is a more common habit in Slovenian population. Each questionnaire item was then evaluated with a score of one to three points in order of levels of habitual PA as described in original paper and added up to determine the total NEAT score. NEAT questionnaire tries to indirectly assess the amount of activity of daily living that is not always perceived as PA in other questionnaires for assessing PA. Its use for this task was validated by triaxial accelerometer in people with type 2 diabetes (Hamasaki et al., 2014). PA assessed by NEAT score partly overlaps with MPA assessed with GPAQ. Nevertheless, GPAQ has higher threshold for detecting PA while including only MPA that causes small increases in breathing or heart rate such as brisk walking (or carrying light loads) and also does not capture PA episodes shorter than $10 \mathrm{~min}$. 
Moreover, participants also reported the frequency of recent hypoglycemia. Frequency of hypoglycemia was defined with participant's self-estimated number of documented hypoglycemias $(<3.9 \mathrm{mmol} / \mathrm{l})$ in the last month.

\section{Statistical Analysis}

Data were analyzed using IBM SPSS 21 (IBM Inc., Chicago, IL, United States). Categorical data are presented as numbers (percentages), and numerical variables are presented as means (standard deviations). All numerical data were screened for normality of distribution using Shapiro-Wilk's test and histograms. Correlations were calculated using Pearson's correlation coefficient for normally distributed and linear correlation, otherwise Spearman's rank correlation coefficient was applied. Additionally, the associations between demographic variables (sex, age, BMI), PA variables (NEAT, MPA, and VPA), and selected metabolic variables $\left(\mathrm{HbA}_{1 c}\right.$, frequency of hypoglycemia), hemodynamics, and blood lipids were determined using the multivariate linear regression, after the data were screened for normality of distribution, homoscedasticity, linearity, and multicollinearity (Field, 2013). In the case of asymmetrically distributed outcome variable, the data were transformed using logarithmic (frequency of hypoglycemia, HDL) or $1 / Y$ transformation (triglycerides, BMI, SBP). The significance level was set at $p$-values $<0.05$.

\section{RESULTS}

A total of 109 people diagnosed with type 1 diabetes [54 men and 55 women, aged 38 (10) years, height $173.7(8.7) \mathrm{cm}$, and weight $77.33(15.70) \mathrm{kg}$ ] were included in the sample (Table 1), with mean type 1 diabetes duration of 22 (10) years. Majority of participants $(65.1 \%)$ were regular users of insulin pump, $31 \%$ of them were also using CGMS. Thirty-five percent of the study participants used MDIs for glucose management, $13.2 \%$ of them used CGMS. Half of them failed to reach the goal for glycemic control [median $\mathrm{HbA}_{1 c} 53.0 \mathrm{mmol} / \mathrm{mol}$ (7.0\%)] and reported more than four events (median 4.0 events per month) of hypoglycemia per month. In addition to therapy for glucose control, some participants had prescribed antihypertensive (15.0\%) and/or medication therapy for dyslipidemia (14.0\%). We observed statistically significant differences in PA between genders, with men spending more time for VPA and women being more active in NEPA. Correlations of different types of PA with different observed parameters of metabolic control are presented in Table 2 and multivariate regression models in Table 3.

\section{Glycemic Control}

There was a significant positive correlation between NEAT and $\mathrm{HbA}_{1 c}$ when calculated for sample $(p=0.021)$ and subsample of men $(p=0.038)$, but not for women. Sub-analysis of people without usage of insulin pump or sensor technologies $(N=30)$ also showed positive correlation between $\mathrm{HbA}_{1 c}$ and NEAT $(r=0.49 ; p=0.006)$. Additionally, a tendency toward significant positive correlation was obtained between $\mathrm{HbA}_{1 c}$ and VPA for the whole sample $(p=0.056)$. Furthermore, we obtained significant regression model explaining $16.8 \%$ of the variance of $\mathrm{HbA}_{1 c}$ predicted from sex, BMI, CGMS use, and NEAT. Higher BMI and NEAT levels were significantly associated with higher levels of $\mathrm{HbA}_{1 c}$, whereas in the same model the use of the CGMS significantly lowered $\mathrm{HbA}_{1 c}$ levels. On the contrary, when NEAT was substituted for MPA or VPA in the regression model, there were no significant associations with $\mathrm{HbA}_{1 c}(\mathrm{MPA} p=0.087$, VPA $p=0.111$ ). Total duration of work as assessed by GPAQ (moderate and vigorous) was significantly associated with $\operatorname{HbA}_{1 c}(p=0.007)$ when adjusted for sex, BMI, and CGMS use, while such associations were not significant with total duration of recreation as assessed by GPAQ $(p=0.443)$. Multivariate regression models predicting frequency of hypoglycemia with sex, $\mathrm{HbA}_{1 c}$, and different types of PA were not significant, although there was a tendency toward significant negative correlation between VPA and frequency of hypoglycemia $(r=-0.201, p=0.050)$.

\section{Body Weight and Blood Pressure}

Negative correlation between NEAT and BMI was obtained when performed for all participants $(r=-0.352, p<0.001)$ and for subsample of men $(r=-0.411, p=0.002)$, also similar significant associations were observed in the multivariate models when controlled for sex, $\mathrm{HbA}_{1 \mathrm{c}}$, and age (Table 3). On the contrary, when NEAT was substituted by VPA and MPA, such associations with $1 / \mathrm{BMI}$ were not significant. There was a significant positive correlation between VPA and SBP when calculated for sample $(p=0.001)$ and sub-sample of women $(p=0.047)$, but not for men. SBP was positively associated with duration of MPA $(p=0.009)$ and VPA $(p=0.012)$, but not with NEAT score $(p=0.830)$ when controlled for sex and BMI, respectively. When the same multivariate models were applied for prediction of DBP, we failed to obtain significant models.

\section{Serum Lipids}

Correlations of serum lipids concentrations and different types of PA were significant only between VPA and total cholesterol $(r=-0.234, p=0.046)$ and LDL cholesterol $(r=-0.249$, $p=0.034)$, respectively. Sub-analysis in men showed significant correlations between VPA and LDL cholesterol $(r=-0.344$, $p=0.028)$ and also between NEAT and triglycerides $(r=0.371$, $p=0.017)$.

When adjusted for sex and BMI, NEAT and VPA explained 23.6 and $17.2 \%$ of $\log \mathrm{HDL}$ variance, respectively. Higher levels of NEAT and VPA were significantly associated with lower levels of $\log \mathrm{HDL}$, resulting in association with higher HDL levels (after the outcome was transformed using the inverse logarithm). When applying the same independent variables for predicting total cholesterol and LDL levels, there was a significant association with sex, BMI, and VPA, explaining $8.6 \%$ of variance of total cholesterol and $9.3 \%$ of LDL levels. Also, regression models adjusted for sex and BMI showed that higher values of VPA were significantly associated with lower levels of total cholesterol $(p=0.009)$ and LDL $(p=0.005)$. 
TABLE 1 | Baseline sample characteristics.

\begin{tabular}{|c|c|c|c|c|c|}
\hline & All & Men & Women & $p$-value & $N$ \\
\hline \multicolumn{6}{|l|}{ Demographics } \\
\hline Gender, men/women $(n)$ & 109 & 54 & 55 & & 109 \\
\hline Age (years) & $38(10)$ & $37(9)$ & $38(10)$ & 0.849 & 108 \\
\hline Height (cm) & $173.72(8.74)$ & $179.43(7.17)$ & $167.69(5.67)$ & $<0.001$ & 105 \\
\hline Weight (kg) & $77.33(15.70)$ & $84.41(13.04)$ & $69.98(14.93)$ & $<0.001$ & 106 \\
\hline $\mathrm{BMI}\left(\mathrm{kg} / \mathrm{m}^{2}\right)$ & $25.54(4.57)$ & $26.22(3.78)$ & $24.83(5.23)$ & 0.008 & 105 \\
\hline \multicolumn{6}{|l|}{ Diabetes characteristics } \\
\hline Insulin pump - n (\%) & $71(65.1)$ & $34(63.0)$ & $37(67.3)$ & 0.690 & 109 \\
\hline CGMS - n (\%) & $27(24.8)$ & $16(29.6)$ & $11(20.0)$ & 0.274 & 109 \\
\hline Duration of diabetes (years) & $22(10)$ & $22(11)$ & $22(10)$ & 0.815 & 102 \\
\hline Frequency of hypoglycemia $(n)$ & $6(4)$ & $6(4)$ & $6(5)$ & 0.888 & 99 \\
\hline \multicolumn{6}{|c|}{ Hemodynamics and blood markers } \\
\hline Systolic blood pressure (mmHg) & $129.64(17.18)$ & $135.81(16.43)$ & $123.10(15.41)$ & $<0.001$ & 105 \\
\hline Diastolic blood pressure (mmHg) & $79.10(8.75)$ & $81.17(8.81)$ & $76.90(8.21)$ & 0.011 & 105 \\
\hline $\mathrm{HbA}_{1 \mathrm{c}}(\mathrm{mmol} / \mathrm{mol})$ & 53.28 (9.69) & $53.86(9.78)$ & $52.69(9.64)$ & 0.346 & 106 \\
\hline $\mathrm{HbA}_{1 \mathrm{c}}(\%)$ & $7.03(0.89)$ & $7.08(0.90)$ & $6.97(0.88)$ & 0.346 & 106 \\
\hline Serum cholesterol (mmol/L) & $4.91(0.73)$ & $4.81(0.66)$ & $5.04(0.80)$ & 0.181 & 75 \\
\hline Serum HDL (mmol/L) & $1.75(0.50)$ & $1.6(0.41)$ & $1.94(0.54)$ & 0.005 & 75 \\
\hline Serum LDL (mmol/L) & $2.77(0.62)$ & $2.84(0.64)$ & $2.66(0.58)$ & 0.195 & 75 \\
\hline Triglycerides (mmol/L) & $0.92(0.45)$ & $0.99(0.56)$ & $0.84(0.25)$ & 0.157 & 75 \\
\hline \multicolumn{6}{|l|}{ Physical activity } \\
\hline MPA (min/week) & $688.2(711.13)$ & $680.80(806.86)$ & $695.38(612.85)$ & 0.180 & 104 \\
\hline VPA (min/week) & $418.16(649.45)$ & 501.47 (696.40) & 336.44 (595.28) & 0.039 & 103 \\
\hline NEAT score (points) & $73.14(9.85)$ & $70.55(10.15)$ & $75.74(8.90)$ & 0.006 & 106 \\
\hline
\end{tabular}

BMI, body mass index; CGMS, continuous glucose monitoring system; HDL, high-density lipoprotein; LDL, Iow-density lipoprotein; MPA, moderate physical activity; VPA, vigorous physical activity; NEAT, non-exercise activity thermogenesis; data are expressed as mean (SD). $p$ value $<0.05$.

\section{DISCUSSION}

Physical activity has a potential to improve glucose metabolism in people with prediabetes and type 2 diabetes (Boniol et al., 2017). This potential is hindered by a challenge of maintaining blood glucose within range with insulin therapy, that is especially demanding for persons with type 1 diabetes, who often do not achieve appropriate chronic glucose control (McCarthy et al., 2016). To maintain appropriate glycemic profile around PA they often have to keep their blood glucose higher, reduce their insulin dose, and/or increase consumption of carbohydrates, especially when PA is not planned in advance (Francescato et al., 2015).

Results of our study failed to demonstrate benefit of LTPA on chronic glycemic control assessed by $\mathrm{HbA}_{1 \mathrm{c}}$ in people with type 1 diabetes. Furthermore, we demonstrated a positive association of NEAT score and total duration of work activity assessed with GPAQ with chronic glycemic control, with no significant association with frequency of self-reported hypoglycemia.

Majority of people with type 1 diabetes do not meet the exercise levels as recommended by guidelines (McCarthy et al., 2016). Csizmadi et al. (2011) observed relatively low levels of leisure time activity among adult Canadian population, with occupational and household activity accounting for $>80 \%$ of overall hours of daily PA. Low intensity PA of daily living constitutes about $20 \%$ of daily energy expenditure
(Manohar et al., 2013) and is usually of longer duration, more frequent, and unpredictable compared to planned exercise. High absolute burden of PA of daily living and work on efforts for self-management of glycemia could be one of the factors for positive association with $\mathrm{HbA}_{1 \mathrm{c}}$. Additionally, reducing inactivity by increasing the time spent walking/standing seems to be more effective in metabolic improvement than $1 \mathrm{~h}$ of physical exercise, when energy expenditure is kept constant (Duvivier et al., 2013).

Physical activity is frequently perceived as a significant risk of harm through hypoglycemia from perspective of people with diabetes (Brazeau et al., 2008). Fear of hypoglycemia is associated with higher $\mathrm{HbA}_{1 \mathrm{c}}$ levels (Ahola et al., 2016) and could be one of the reasons for higher personal glycemic targets with insulin therapy while coping with daily glycemic imbalance associated with PA. $\mathrm{HbA}_{1 \mathrm{c}}$ is not the only indicator of glycemic control, because dynamic in glycemic variability may not be reflected in this measure (Suh and Kim, 2015). Data on influence of chronic effects of exercise training on glucose variability are still scarce, even if it represents one of the most robust predictors of hypoglycemia (Klaprat et al., 2019). One of the promising options for managing degree of glucose variability associated with PA are emerging modern technologies (sensors with adequate accuracy for exercise, modern insulin pumps, and improvement in insulin analogs) (Houlder and Yardley, 2018), while their use and association with NEAT still requires further studies for clarification. 
TABLE 2 | Correlations of PA types with clinical parameters and biomarkers.

\begin{tabular}{|c|c|c|c|c|c|c|c|c|}
\hline \multirow[b]{2}{*}{ Parameter } & \multirow[b]{2}{*}{ PA } & \multirow[b]{2}{*}{$N$} & \multicolumn{2}{|c|}{ All } & \multicolumn{2}{|c|}{ Men } & \multicolumn{2}{|c|}{ Women } \\
\hline & & & $r$ & $p$-value & $r$ & $p$-value & $r$ & $p$-value \\
\hline \multirow[t]{3}{*}{$\mathrm{HbA}_{1 \mathrm{c}}$} & VPA & 102 & 0.190 & 0.056 & 0.094 & 0.513 & 0.228 & 0.107 \\
\hline & MPA & 103 & 0.044 & 0.660 & -0.014 & 0.925 & 0.095 & 0.503 \\
\hline & NEAT & 105 & $0.226^{\ddagger}$ & 0.021 & $0.286^{\ddagger}$ & 0.038 & 0.152 & 0.281 \\
\hline \multirow[t]{3}{*}{ Hypoglycemia } & VPA & 96 & -0.201 & 0.050 & -0.224 & 0.118 & -0.202 & 0.178 \\
\hline & MPA & 96 & -0.023 & 0.827 & -0.055 & 0.708 & -0.025 & 0.865 \\
\hline & NEAT & 98 & -0.165 & 0.105 & -0.120 & 0.401 & -0.276 & 0.060 \\
\hline \multirow[t]{3}{*}{ SBP } & VPA & 101 & 0.320 & 0.001 & 0.211 & 0.137 & 0.282 & 0.047 \\
\hline & MPA & 102 & 0.169 & 0.089 & 0.211 & 0.137 & 0.268 & 0.057 \\
\hline & NEAT & 104 & -0.150 & 0.127 & $-0.273^{\ddagger}$ & 0.048 & 0.057 & 0.691 \\
\hline \multirow[t]{3}{*}{$\mathrm{DBP}$} & VPA & 101 & 0.056 & 0.575 & -0.016 & 0.914 & 0.048 & 0.740 \\
\hline & MPA & 102 & -0.017 & 0.866 & 0.007 & 0.960 & 0.033 & 0.819 \\
\hline & NEAT & 104 & $-0.124^{\ddagger}$ & 0.211 & $-0.116^{\ddagger}$ & 0.408 & $-0.006^{\ddagger}$ & 0.968 \\
\hline \multirow[t]{3}{*}{ BMl } & VPA & 101 & -0.008 & 0.934 & 0.002 & 0.988 & -0.071 & 0.622 \\
\hline & MPA & 102 & -0.175 & 0.079 & -0.112 & 0.431 & -0.167 & 0.241 \\
\hline & NEAT & 104 & -0.352 & 0.000 & -0.411 & 0.002 & -0.202 & 0.154 \\
\hline \multirow[t]{3}{*}{ Cholesterol } & VPA & 73 & -0.234 & 0.046 & -0.240 & 0.130 & -0.150 & 0.412 \\
\hline & MPA & 72 & -0.123 & 0.303 & -0.093 & 0.575 & -0.144 & 0.424 \\
\hline & NEAT & 74 & $-0.203^{\ddagger}$ & 0.082 & $-0.278^{\ddagger}$ & 0.078 & $-0.252^{\ddagger}$ & 0.157 \\
\hline \multirow[t]{3}{*}{ LDL } & VPA & 73 & -0.249 & 0.034 & -0.344 & 0.028 & -0.261 & 0.149 \\
\hline & MPA & 72 & -0.111 & 0.354 & -0.104 & 0.530 & -0.047 & 0.796 \\
\hline & NEAT & 74 & $-0.221^{\ddagger}$ & 0.058 & $-0.228^{\ddagger}$ & 0.151 & $-0.128^{\ddagger}$ & 0.478 \\
\hline \multirow[t]{3}{*}{$\mathrm{HDL}$} & VPA & 72 & -0.071 & 0.550 & 0.158 & 0.324 & -0.093 & 0.612 \\
\hline & MPA & 71 & -0.062 & 0.606 & -0.083 & 0.613 & -0.148 & 0.413 \\
\hline & NEAT & 73 & -0.112 & 0.341 & $-0.158^{\ddagger}$ & 0.323 & $-0.328^{\ddagger}$ & 0.062 \\
\hline \multirow[t]{3}{*}{ Triglycerides } & VPA & 73 & -0.036 & 0.764 & -0.077 & 0.632 & 0.029 & 0.874 \\
\hline & MPA & 72 & 0.123 & 0.302 & 0.173 & 0.293 & 0.014 & 0.940 \\
\hline & NEAT & 74 & 0.174 & 0.138 & 0.371 & 0.017 & -0.119 & 0.511 \\
\hline
\end{tabular}

¡Pearson's correlation coefficient. r, Spearman's correlation coefficient; SBP, systolic blood pressure; DBP, diastolic blood pressure; BMI, body mass index; HDL, high-

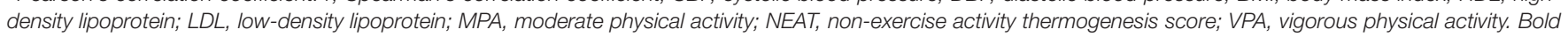
values indicate significant at $p<0.05$.

Large proportion of people with type 1 diabetes is obese or overweight (McCarthy et al., 2016), which is in line with results from our sample. Emerging evidence suggests that obesity contributes to insulin resistance, dyslipidemia, and cardiometabolic complications in type 1 diabetes, while prevalence of obesity increases with alarming rate in this population (Corbin et al., 2018). As expected from previous studies that emphasize the role of NEAT in preventing obesity (Ravussin, 2005), our results also suggest association of higher NEAT score with lower BMI in people with type 1 diabetes. Recent study by $\mathrm{Du}$ et al. (2019) shows a worrying trend in PA among US adults in years 20072016, with no improvement in duration of aerobic activity and statistically significant increase of sedentary behavior assessed by GPAQ. Recommendations on PA for weight management have traditionally been focused on leisure time activity, while other PA domains also offer many opportunities for interventions (Ravussin, 2005). Furthermore, there is a possible role of NEAT in behavioral compensation mechanisms after PA interventions that still awaits further clarification (Silva et al., 2018).

Predominant form of PA in people with type 2 diabetes are different domestic chores (Cloix et al., 2015). It is therefore not surprising that NEAT score seems to be associated with amelioration in insulin sensitivity, waist circumference, HDL, blood pressure, and the markers for atherosclerosis in people with type 2 diabetes without hypoglycemic therapy (Hamasaki et al., 2013). Limited research in type 1 diabetes suggests that interventions with exercise programs improve lipid levels, endothelial function, and insulin resistance but not blood pressure (Chimen et al., 2012). Exercise training does not significantly change resting SBP according to the recent metaanalysis (Ostman et al., 2018). Study of Bohn et al. (2015) did find an inverse association between LTPA and DBP without significant 
TABLE 3 | Multivariate regression models.

\begin{tabular}{|c|c|c|c|c|c|}
\hline \multirow[t]{2}{*}{ Dependent variable } & \multirow[t]{2}{*}{ Predictors } & \multicolumn{3}{|c|}{ Multivariate regression model } & \multirow[t]{2}{*}{ Adjusted $R^{2}$ (model $p$-value) } \\
\hline & & Coefficient & $95 \% \mathrm{Cl}$ & $p$-value & \\
\hline \multirow[t]{4}{*}{ 1/BMl } & Sex & -0.00159 & $-0.00401,0.00083$ & 0.194 & $0.165(<0.001)$ \\
\hline & Age & -0.00013 & $-0.00024,-0.00001$ & 0.035 & \\
\hline & $\mathrm{HbA}_{1 \mathrm{c}}$ & -0.00176 & $-0.00310,-0.00042$ & 0.011 & \\
\hline & NEAT & 0.00021 & $0.00008,0.00034$ & 0.001 & \\
\hline \multirow[t]{4}{*}{$\mathrm{HbA}_{1 \mathrm{c}}$} & Sex & 0.226 & $-0.104,0.557$ & 0.177 & $0.168(<0.001)$ \\
\hline & CGMS use & -0.455 & $-0.826,-0.084$ & 0.017 & \\
\hline & $\mathrm{BMI}$ & 0.063 & $0.027,0.099$ & $<0.001$ & \\
\hline & NEAT & 0.030 & $0.013,0.047$ & $<0.001$ & \\
\hline \multirow[t]{4}{*}{$\mathrm{HbA}_{1 \mathrm{c}}$} & Sex & -0.0054 & -0.3344 .0 .3236 & 0.974 & $0.130(0.001)$ \\
\hline & CGMS use & -0.3524 & $-0.7384,0.0336$ & 0.073 & \\
\hline & $\mathrm{BMI}$ & 0.0533 & $0.0172,0.0893$ & 0.004 & \\
\hline & WORK & 0.0002 & $0.0001,0.0004$ & 0.007 & \\
\hline \multirow[t]{4}{*}{$\mathrm{HbA}_{1 \mathrm{c}}$} & Sex & 0.1435 & $-0.2041,0.4911$ & 0.415 & $0.075(0.021)$ \\
\hline & CGMS use & -0.4442 & $-0.8406,-0.0479$ & 0.028 & \\
\hline & $\mathrm{BMI}$ & 0.0519 & $0.0136,0.0902$ & 0.009 & \\
\hline & LEISURE & -0.0002 & $-0.0008,0.0004$ & 0.443 & \\
\hline \multirow[t]{3}{*}{ 1/SBP } & Sex & -0.00061 & $-0.00096,-0.00026$ & $<0.001$ & $0.306(<0.001)$ \\
\hline & $\mathrm{BMl}$ & -0.00010 & $-0.00014,-0.00006$ & $<0.001$ & \\
\hline & NEAT & 0.000002 & $-0.00002,0.00002$ & 0.830 & \\
\hline \multirow[t]{3}{*}{ 1/SBP } & Sex & -0.00063 & $-0.00096,-0.00030$ & $<0.001$ & $0.348(<0.001)$ \\
\hline & $\mathrm{BMI}$ & -0.00011 & $-0.00015,-0.00007$ & $<0.001$ & \\
\hline & MPA & -0.0000003 & $-0.00000,-0.00000$ & 0.009 & \\
\hline \multirow[t]{3}{*}{ 1/SBP } & Sex & -0.000603 & $-0.00094,-0.00027$ & $<0.001$ & $0.349(<0.001)$ \\
\hline & $\mathrm{BMl}$ & -0.000099 & $-0.00014,-0.00006$ & $<0.001$ & \\
\hline & VPA & -0.0000003 & $-0.000001,-0.00000$ & 0.012 & \\
\hline \multirow[t]{3}{*}{ Cholesterol } & Sex & -0.230 & $-0.565,0.106$ & 0.177 & $0.086(0.027)$ \\
\hline & $\mathrm{BMl}$ & 0.00812 & $-0.026,0.042$ & 0.637 & \\
\hline & VPA & -0.00034 & $-0.001,-0.00009$ & 0.009 & \\
\hline \multirow[t]{3}{*}{ LDL } & Sex & 0.17110 & $-0.11030,0.45250$ & 0.229 & $0.093(0.021)$ \\
\hline & $\mathrm{BMI}$ & 0.01087 & $-0.01783,0.03956$ & 0.452 & \\
\hline & VPA & -0.0003 & $-0.00052,-0.00001$ & 0.005 & \\
\hline \multirow[t]{3}{*}{$\log (H D L)$} & Sex & -0.101 & $-0.154,-0.047$ & $<0.001$ & $0.236(<0.001)$ \\
\hline & $\mathrm{BMl}$ & -0.008 & $-0.014,-0.003$ & 0.002 & \\
\hline & NEAT & -0.004 & $-0.006,-0.001$ & 0.008 & \\
\hline \multirow[t]{3}{*}{$\log (\mathrm{HDL})$} & Sex & -0.078 & $-0.133,-0.022$ & 0.007 & $0.134(0.006)$ \\
\hline & $\mathrm{BMl}$ & -0.007 & $-0.012,-0.001$ & 0.021 & \\
\hline & MPA & -0.000008 & $-0.00004,0.00002$ & 0.607 & \\
\hline \multirow[t]{3}{*}{$\log (\mathrm{HDL})$} & Sex & -0.072 & $-0.124,-0.019$ & 0.009 & $0.172(0.001)$ \\
\hline & $\mathrm{BMl}$ & -0.006 & $-0.012,-0.001$ & 0.021 & \\
\hline & VPA & -0.000041 & $-0.00008,-0.000001$ & 0.043 & \\
\hline
\end{tabular}

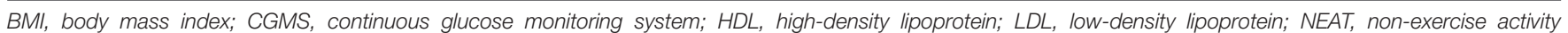
thermogenesis score; MPA, moderate physical activity; VPA, vigorous physical activity; LEISURE, total leisure PA; WORK, total work-related PA.

association with SBP. Surprisingly, we have observed a weak positive association of VPA and MPA with SBP in our sample when adjusted for sex and BMI, while we could not confirm such association with NEAT score. As expected from previous studies, our results also show positive association of NEAT score and VPA with HDL levels. Furthermore, we observed negative association 
of VPA with total and LDL cholesterol when adjusted for sex and BMI.

Because of relatively large sample size of our study, we could detect weak to moderate associations of sub-types of NEAT with different risk factors, which may have clinical implications in the population that has a known elevated risk for cardiovascular disease and sub-optimal risk factor control (McCarthy et al., 2016). Furthermore, frequent LTPA seems to reduce the risk of CVD events in people with type 1 diabetes in addition to traditional risk factors (Tikkanen-Dolenc et al., 2017). Exercise has also been shown to improve immune system function, body composition, physiological well-being, cardiovascular fitness, and late complications of diabetes in people with type 1 diabetes (Codella et al., 2017; Kluding et al., 2017). Further studies in type 1 diabetes are needed to evaluate the role of NEAT beyond traditional risk factors.

Our study has some important limitations, most important are cross-sectional design of the study and unspecific method (questionnaire) for estimating amount of NEAT. The validity of methods for measuring NEAT is still controversial. However, some authors believe that a questionnaire is one of the most useful and reliable methods of measuring NEAT at the moment (Hamasaki et al., 2013). Nevertheless, there is a need for use and further development of more precise methods for estimating NEAT (Manohar et al., 2013; Zecchin et al., 2013). Additional studies are also needed to exclude possible confounding variables (influence of diet, sociocultural background, and other factors) that may influence results of our analysis because of crosssectional design of the study.

\section{CONCLUSION}

Higher NEAT score is associated with lower body weight and higher HDL in adult people with type 1 diabetes, but may also present an additional burden for them with more challenging environment regarding glycemic control. New strategies for people with type 1 diabetes with higher levels of NEAT should

\section{REFERENCES}

Ahola, A. J., Saraheimo, M., Freese, R., Mäkimattila, S., Forsblom, C., Groop, P.-H., et al. (2016). Fear of hypoglycaemia and self-management in type 1 diabetes. J. Clin. Transl. Endocrinol. 4, 13-18. doi: 10.1016/j.jcte.2016.02.002

Armstrong, T., and Bull, F. (2006). Development of the world health organization global physical activity questionnaire (GPAQ). J. Public Health 14, 66-70. doi: 10.1007/s10389-006-0024-X

Balk, S. N., Schoenaker, D. A., Mishra, G. D., Toeller, M., Chaturvedi, N., Fuller, J. H., et al. (2016). Association of diet and lifestyle with glycated haemoglobin in type 1 diabetes participants in the EURODIAB prospective complications study. Eur. J. Clin. Nutr. 70, 229-236. doi: 10.1038/ejcn.2015.110

Bohn, B., Herbst, A., Pfeifer, M., Krakow, D., Zimny, S., Kopp, F., et al. (2015). Impact of physical activity on glycemic control and prevalence of cardiovascular risk factors in adults with type 1 diabetes: a cross-sectional multicenter study of 18,028 patients. Diabetes Care 38, 1536-1543. doi: 10.2337/dc1 5-0030

Boniol, M., Dragomir, M., Autier, P., and Boyle, P. (2017). Physical activity and change in fasting glucose and HbAlc: a quantitative meta-analysis of randomized trials. Acta Diabetol. 54, 983-991. doi: 10.1007/s00592-017-1037-3 be developed in the future to strengthen the protective effect of NEPA with combating modern sitting environment and also tackling the problem of the potentially greater glucose variability. Methods for precise estimation of NEAT should be further developed because of important role of this "silent" part of energy expenditure in people with type 1 diabetes, that is often overlooked in research and also in clinical practice.

\section{DATA AVAILABILITY STATEMENT}

The raw data supporting the conclusions of this manuscript will be made available by the authors, without undue reservation, to any qualified researcher.

\section{ETHICS STATEMENT}

The studies involving human participants were reviewed and approved by the National Medical Ethics Committee of Slovenia (Ref. No. 0120-258/2017/4). The patients/participants provided their written informed consent to participate in this study.

\section{AUTHOR CONTRIBUTIONS}

IS, AZ, and VH created the study conception and design. AZ and IS were involved in the data collection. TK, IS, and AZ conducted the data analysis and wrote the manuscript. All authors were involved in drafting and revising the manuscript.

\section{FUNDING}

This work was financially supported by the University Medical Centre Ljubljana to cover the costs of publication. TK received the research fellowship from the Slovenian Research Agency for Ph.D. studies (Grant No. 630-72/2019-1).

Brazeau, A.-S., Rabasa-Lhoret, R., Strychar, I., and Mircescu, H. (2008). Barriers to physical activity among patients with type 1 diabetes. Diabetes Care 31, 2108-2109. doi: 10.2337/dc08-0720

Chimen, M., Kennedy, A., Nirantharakumar, K., Pang, T. T., Andrews, R., and Narendran, P. (2012). What are the health benefits of physical activity in type 1 diabetes mellitus? A literature review. Diabetologia 55, 542-551. doi: 10.1007/ s00125-011-2403-2

Cloix, L., Caille, A., Helmer, C., Bourdel-Marchasson, I., Fagot-Campagna, A., Fournier, C., et al. (2015). Physical activity at home, at leisure, during transportation and at work in French adults with type 2 diabetes: the ENTRED physical activity study. Diabetes Metab. 41, 37-44. doi: 10.1016/j.diabet.2014. 07.003

Codella, R., Terruzzi, I., and Luzi, L. (2017). Why should people with type 1 diabetes exercise regularly? Acta Diabetol. 54, 615-630. doi: 10.1007/s00592017-0978-x

Corbin, K. D., Driscoll, K. A., Pratley, R. E., Smith, S. R., Maahs, D. M., and MayerDavis, E. J. (2018). Obesity in type 1 diabetes: pathophysiology, clinical impact, and mechanisms. Endocr. Rev. 39, 629-663. doi: 10.1210/er.2017-00191

Csizmadi, I., Lo Siou, G., Friedenreich, C. M., Owen, N., and Robson, P. J. (2011). Hours spent and energy expended in physical activity domains: results from 
the tomorrow projectcohort in Alberta, Canada. Int. J. Behav. Nutr. Phys. Act. 8:110. doi: 10.1186/1479-5868-8-110

Ding, S., and Schumacher, M. (2016). Sensor monitoring of physical activity to improve glucose management in diabetic patients: a review. Sensors 16:E589. doi: 10.3390/s16040589

Du, Y., Liu, B., Sun, Y., Snetselaar, L. G., Wallace, R. B., and Bao, W. (2019). Trends in adherence to the physical activity guidelines for Americans for Aerobic activity and time spent on sedentary behavior among US adults, 2007 to 2016. JAMA Netw. Open 2:e197597. doi: 10.1001/jamanetworkopen.2019.7597

Dunstan, D. W., Kingwell, B. A., Larsen, R., Healy, G. N., Cerin, E., Hamilton, M. T., et al. (2012). Breaking up prolonged sitting reduces postprandial glucose and insulin responses. Diabetes Care 35, 976-983. doi: 10.2337/dc11-1931

Duvivier, B. M., Schaper, N. C., Bremers, M. A., van Crombrugge, G., Menheere, P. P., Kars, M., et al. (2013). Minimal intensity physical activity (standing and walking) of longer duration improves insulin action and plasma lipids more than shorter periods of moderate to vigorous exercise (cycling) in sedentary subjects when energy expenditure is comparable. PLoS One 8:e55542. doi: 10. 1371/journal.pone.0055542

Farabi, S. S., Carley, D. W., Cinar, A., and Quinn, L. (2015). Routine daily physical activity and glucose variations are strongly coupled in adults with T1DM. Physiol. Rep. 3:e12644. doi: 10.14814/phy2.12644

Field, A. (2013). Discovering Statistics using IBM SPSS Statistics. London: SAGE.

Francescato, M. P., Stel, G., Stenner, E., and Geat, M. (2015). Prolonged exercise in type 1 diabetes: performance of a customizable algorithm to estimate the carbohydrate supplements to minimize glycemic imbalances. PLoS One 10:e0125220. doi: 10.1371/journal.pone.0125220

Hamasaki, H., Yanai, H., Kakei, M., Noda, M., and Ezaki, O. (2014). The validity of the non-exercise activity thermogenesis questionnaire evaluated by objectively measured daily physical activity by the triaxial accelerometer. BMC Sports Sci. Med. Rehabil. 6:27. doi: 10.1186/2052-1847-6-27

Hamasaki, H., Yanai, H., Mishima, S., Mineyama, T., Yamamoto-Honda, R., Kakei, M., et al. (2013). Correlations of non-exercise activity thermogenesis to metabolic parameters in Japanese patients with type 2 diabetes. Diabetol. Metab. Syndr. 5:26. doi: 10.1186/1758-5996-5-26

Houlder, S. K., and Yardley, J. E. (2018). Continuous glucose monitoring and exercise in type 1 diabetes: past, present and future. Biosensors 8:E73. doi: 10.3390/bios 8030073

Klaprat, N., MacIntosh, A., and McGavock, J. M. (2019). Gaps in knowledge and the need for patient-partners in research related to physical activity and type 1 diabetes: a narrative review. Front. Endocrinol. 10:42. doi: 10.3389/fendo.2019. 00042

Kluding, P. M., Bareiss, S. K., Hastings, M., Marcus, R. L., Sinacore, D. R., and Mueller, M. J. (2017). Physical training and activity in people with diabetic peripheral neuropathy: paradigm shift. Phys. Ther. 97, 31-43. doi: 10.2522/ptj. 20160124

Levine, J. A. (2004). Nonexercise activity thermogenesis (NEAT): environment and biology. Am. J. Physiol. Endocrinol. Metab. 286, E675-E685.

Levine, J. A. (2015). Sick of sitting. Diabetologia 58, 1751-1758. doi: 10.1007/ s00125-015-3624-6

Manohar, C., Levine, J. A., Nandy, D. K., Saad, A., Dalla Man, C., McCrady-Spitzer, S. K., et al. (2012). The effect of walking on postprandial glycemic excursion in patients with type 1 diabetes and healthy people. Diabetes Care 35, 2493-2499. doi: $10.2337 / \mathrm{dc} 11-2381$

Manohar, C., O’Keeffe, D. T., Hinshaw, L., Lingineni, R., McCrady-Spitzer, S. K., Levine, J. A., et al. (2013). Comparison of physical activity sensors and heart rate monitoring for real-time activity detection in type 1 diabetes and control subjects. Diabetes Technol. Ther. 15, 751-757. doi: 10.1089/dia.2013.0044

McCarthy, M. M., Funk, M., and Grey, M. (2016). Cardiovascular health in adults with type 1 diabetes. Prev. Med. 91, 138-143. doi: 10.1016/j.ypmed.2016.08.019

Ogata, H., Nakamura, K., Sato, M., Tokuyama, K., Nagasaka, S., Ebine, N., et al. (2013). Lack of negative correlation in glucose dynamics by nonexercise activity thermogenesis restriction in healthy adults. Med. Sci. Sports Exerc. 45, 60-66. doi: 10.1249/MSS.0b013e31826c2dfc

Ostman, C., Jewiss, D., King, N., and Smart, N. A. (2018). Clinical outcomes to exercise training in type 1 diabetes: a systematic review and meta-analysis. Diabetes Res. Clin. Pract. 139, 380-391. doi: 10.1016/j.diabres.2017.11.036

Ravussin, E. (2005). A NEAT way to control weight? Science 307, 530-531. doi: $10.1126 /$ science. 1108597

Riddell, M. C., Gallen, I. W., Smart, C. E., Taplin, C. E., Adolfsson, P., Lumb, A. N., et al. (2017). Exercise management in type 1 diabetes: a consensus statement. Lancet Diabetes Endocrinol. 5, 377-390. doi: 10.1016/S2213-8587(17)30014-1

Silva, A. M., Júdice, P. B., Carraça, E. V., King, N., Teixeira, P. J., and Sardinha, L. B. (2018). What is the effect of diet and/or exercise interventions on behavioural compensation in non-exercise physical activity and related energy expenditure of free-living adults? A systematic review. Br. J. Nutr. 119, 1327-1345. doi: 10.1017/S000711451800096X

Suh, S., and Kim, J. H. (2015). Glycemic variability: how do we measure it and why is it important? Diabetes Metab. J. 39, 273-282. doi: 10.4093/dmj.2015.39.4.273

Tikkanen-Dolenc, H., Wadén, J., Forsblom, C., Harjutsalo, V., Thorn, L. M., Saraheimo, M., et al. (2017). Frequent and intensive physical activity reduces risk of cardiovascular events in type 1 diabetes. Diabetologia 60, 574-580. doi: $10.1007 / \mathrm{s} 00125-016-4189-8$

Wadén, J., Tikkanen, H., Forsblom, C., Fagerudd, J., Pettersson-Fernholm, K., Lakka, T., et al. (2005). Leisure time physical activity is associated with poor glycemic control in type 1 diabetic women: the finndiane study. Diabetes Care 28, 777-782. doi: 10.2337/diacare.28.4.777

Zecchin, C., Facchinetti, A., Sparacino, G., Dalla Man, C., Manohar, C., Levine, J. A., et al. (2013). Physical activity measured by physical activity monitoring system correlates with glucose trends reconstructed from continuous glucose monitoring. Diabetes Technol. Ther. 15, 836-844. doi: 10.1089/dia.2013. 0105

Conflict of Interest: The authors declare that the research was conducted in the absence of any commercial or financial relationships that could be construed as a potential conflict of interest.

Copyright (C) 2019 Štotl, Kambič, Hadžić and Zdolšek. This is an open-access article distributed under the terms of the Creative Commons Attribution License (CC BY). The use, distribution or reproduction in other forums is permitted, provided the original author(s) and the copyright owner(s) are credited and that the original publication in this journal is cited, in accordance with accepted academic practice. No use, distribution or reproduction is permitted which does not comply with these terms. 